\title{
Npr1-regulated gene pathways contributing to cardiac hypertrophy and fibrosis
}

\author{
Leigh J Ellmers ${ }^{1}$, Nicola J A Scott ${ }^{1}$, Jarkko Piuhola ${ }^{1,2}$, Nobuyo Maeda ${ }^{3}$, Oliver Smithies ${ }^{3}$, \\ Chris M Frampton ${ }^{1}$, A Mark Richards ${ }^{1}$ and Vicky A Cameron ${ }^{1}$ \\ ${ }^{1}$ Christchurch Cardioendocrine Research Group, Department of Medicine, Christchurch School of Medicine and Health Sciences, PO Box 4345, Christchurch, New Zealand \\ ${ }^{2}$ Department of Pharmacology and Toxicology, Biocenter Oulu, University of Oulu, 90014 Oulu, Finland \\ ${ }^{3}$ Department of Pathology and Laboratory Medicine, University of North Carolina, Chapel Hill, North Carolina 27599-7525, USA \\ (Requests for offprints should be addressed to L J Ellmers; Email: leigh.ellmers @ chmeds.ac.nz)
}

\begin{abstract}
The natriuretic peptides, atrial (ANP) and brain natriuretic peptide (BNP) are known to suppress cardiac hypertrophy and fibrosis. Both ANP and BNP exert their bioactivities through the Npr1 receptor, and Npr1 knockout mice (Npr1-/-) exhibit marked cardiac hypertrophy and fibrosis. In this study, we investigated which genes within the hypertrophic and fibrotic pathways are influenced by the lack of Npr1 signalling. cDNA microarray and quantitative real-time PCR (RT-PCR) analyses were performed on cardiac ventricles from Npr1-/ - mice. Gene expression at early and late stages during development of hypertrophy was investigated in male and female Npr1 - / - mice at 8 weeks and 6 month of age. Heart weight to body weight ratios $(\mathrm{HW}: \mathrm{BW})$ were maximally increased in 8-week males $(P<0 \cdot 01)$, whilst $\mathrm{HW}$ :BW in females continued to increase progressively up to 6 months $(P<0 \cdot 01)$. This was despite blood pressure being similarly elevated at both the ages in male and female knockout when compared with wild-type (WT) mice $(P<0.001)$. Microarray analysis identified altered gene expression at the earliest steps in the hypertrophy-signalling cascade in Npr1-/- mice, particularly calcium-calmodulin signalling and ion channels, with subsequent changes in the expression of intracellular messengers including protein kinases and transcription factors. Real-time PCR analysis confirmed significant differences in gene expression of ANP, BNP, calmodulin 1, histone deacetylase 7a (HDAC7a), protein kinase C (PKC) เ, (GATA) 4, collagen 1, phospholamban and transforming growth factor- $\beta 1$ in Npr1-/- mice when compared with WT $(P<0.05)$. The present study implicates the calmodulin-CaMK-Hdac-Mef2 and PKC-MAPK-GATA4 pathways in Npr1 mediation of cardiac hypertrophy.
\end{abstract}

Journal of Molecular Endocrinology (2007) 38, 245-257

\section{Introduction}

The term cardiac remodelling is used to describe the changes in size, shape and function of the heart observed during the development of cardiac disease, and is characterised not only by cardiomyocyte hypertrophy and cell death, but also by cardiac fibroblast proliferation and fibrosis (Swynghedauw 1999). Cardiac hypertrophy occurs when terminally differentiated cardiac myocytes respond to an increase in workload or injury (such as hypertension or myocardial infarction) by increasing cell size to maintain a normal cardiac output.

The cardioprotective hormones, the natriuretic peptides, regulate cardiac remodelling by inhibiting both myocyte hypertrophy and cardiac fibrosis (Cao \& Gardner 1995, Horio et al. 2000, Ogawa et al. 2001). Atrial natriuretic peptide (ANP) and brain natriuretic peptide (BNP) are secreted predominantly from the cardiac atria and the ventricles respectively in response to increased cardiac stretch, and regulate blood pressure through their combined actions on vasculature, kidneys and adrenal glands. The actions of both these peptides are mediated by the natriuretic peptide receptor-A (NPR-A or Npr1), a guanylyl cyclase-linked receptor whose activation generates the second messenger cyclic guanosine monophosphate (cGMP). This intracellular signal elicits the well-characterised hypotensive, diuretic and natriuretic effects of these peptides (Espiner et al. 1995).

Evidence for a role of the natriuretic peptides in suppressing cardiac remodelling is supported by both in vitro and in vivo data. Hypertrophy of cardiac myocytes in culture is inhibited by ANP (Horio et al. 2000). Gene delivery of ANP is reported to attenuate hypertension and cardiac hypertrophy in a salt-sensitive rat model (Lin et al. 1998). In addition, all the three members of the natriuretic peptide family, ANP, BNP and C-type natriuretic peptide (CNP), inhibit DNA synthesis in cultured fibroblasts (Cao \& Gardner 1995). The lack of ANP and BNP bioactivities in Nprl gene knockout (Npr1-/-) mice leads to both cardiac hypertrophy and fibrosis (Oliver et al. 1997). The Npr1signalling pathway is reported to directly oppose the hypertrophic response, an effect that is independent of 
the elevated blood pressure observed in these mice (Oliver et al. 1997, Knowles et al. 2001). When blood pressures in Npr1-/- mice were maintained within the normal range by antihypertensive drugs, their cardiac hypertrophy was not ameliorated (Knowles et al. 2001). Furthermore, transgenic mice with the $\mathrm{Nprl}$ gene deletion targeted specifically to cardiac tissue exhibited cardiac hypertrophy in the absence of systemic hypertension (Holtwick et al. 2003), demonstrating conclusively that Npr1-signalling functions as an intrinsic inhibitor of myocyte growth.

Cardiac hypertrophy and fibrosis in Npr $1-/-$ mice have been partially ascribed to activation of the angiotensin 1 receptor (AT1R; Li et al. 2002, 2004). The calcineurin-nuclear factor of activated $\mathrm{T}$ cells (NFAT) pathway mediates AT1R signalling and has also been implicated since blockade of calcineurin activation significantly ameliorated the cardiac hypertrophy and the activation of cardiac gene expression in Npr1 knockout mice (Tokudome et al. 2005). This study aimed to identify the global changes in gene expression that contribute to the hypertrophic and fibrotic pathways influenced by the lack of Npr1 signalling, using the methods of cDNA microarray and quantitative real-time PCR (RT-PCR) analysis of cardiac ventricles from Nprl-/- mice. The influence on gene expression at early and late stages during the development of hypertrophy and fibrosis was investigated in hearts collected from male and female Npr1-/- mice at 8 weeks and 6 months of age. Isolated perfused heart studies were also performed to investigate the contractile response to elevated ventricular stretch in wild type (WT) and Npr1-/- knockout mice associated with development of hypertrophy.

\section{Materials and methods}

\section{Npr1-I- mice}

The original heterozygote breeding stock was kindly donated by Professor Oliver Smithies, Department of Pathology and Laboratory Medicine, University of North Carolina, Chapel Hill, NC, USA. Mouse experiments were performed on Npr1-/ - and WT control mice backcrossed at least 15 generations to $\mathrm{C} 57 \mathrm{BL} / 6$ mice derived from the original mutants, as described previously (Oliver et al. 1997). Experiments were performed according to the protocols approved by the Animal Ethics Committee of the University of Otago.

Adult male and female Npr $1-/-$ and WT $(n=6$ per group) at 8 weeks and 6 months of age were used in this study. Blood pressures were measured on conscious mice by a non-invasive computerised tail cuff system (ADInstruments, Dunedin, New Zealand). The mice were familiarised with being placed in the restrainer and the tail cuff system over 7 days of training, after which daily blood pressure measurements were made for each animal (mean of at least five recordings), and were repeated in subsequent sessions on each of 5 days.

For cardiac gene expression studies, mice were euthanised with an anaesthetic overdose (Halothane; Merial Australia Pty Ltd, Sydney, Australia) before cervical dislocation, hearts were rapidly excised, the atria dissected from the ventricles, weighed and immediately snap-frozen in liquid nitrogen for RNA isolation. The mice were weighed prior to killing to allow heart weight to body weight ratios to be calculated.

\section{RNA isolation}

For each sample, total ventricular RNA was isolated by automated grinding in a Retsch MM301 tissue mill at $30 \mathrm{~Hz}$ for $10 \mathrm{~min}$ in $800 \mu \mathrm{l}$ pre-chilled TRIzol (Invitrogen). Chloroform $(160 \mu \mathrm{l})$ was added and samples were centrifuged at $12000 \mathrm{~g}$ for $15 \mathrm{~min}$. The RNAcontaining supernatant was purified by RNeasy Midi Columns (Qiagen).

\section{cDNA microarray analysis}

Mouse 22K Compugen oligonucleotide microarray slides used in this study (purchased from The Clive and Vera Ramaciotti Centre for Gene Function Analysis, The University of New South Wales, Australia, http:/ /www.ramaciotti.unsw.edu.au), consisted of 22464 60mer oligonucleotides spotted onto GeneMachines OmniGrid Epoxy coated glass slides.

A reference design (Simon \& Dobbin 2003) was employed in the microarray study in which each heart RNA sample was compared with a common reference RNA pool. This allowed the hybridisation intensity for a sample (WTor Npr gene knockout (KO)) to be measured relative to an identical reference sample in all arrays. The reference RNA used in this study was pooled from Npr1 heterozygote $(\mathrm{Npr} 1+/-)$ atria and ventricle tissue of mice ranging in age from 8-week- to 1 year-old.

cDNA probes were generated for each Nprl WT and KO RNA sample of each gender at each age $(n=6$ per group) and also for the reference RNA sample, using the Superscript III Indirect cDNA labelling System (Invitrogen) according to the manufacturer's instructions. For each sample, $10 \mu \mathrm{g}$ total ventricle RNA were transcribed. Each cDNA probe (sample or reference) was resuspended in $2 \mu \mathrm{l} 10 \mathrm{mM}$ EDTA before being combined and denatured at $95{ }^{\circ} \mathrm{C}$ for $10 \mathrm{~min}$. After incubating on ice for $1 \mathrm{~min}$, the probe mix was combined with $70 \mu \mathrm{l}$ pre-heated $\left(65^{\circ} \mathrm{C}\right)$ Slidehybe-1 hybridisation solution (Ambion, Austin, TX, USA) and pipetted onto the microarray slide. The oligonucleotide array slides were pre-treated prior to hybridisation by 
washing in $0 \cdot 1 \%$ SDS at $95{ }^{\circ} \mathrm{C}, 5 \%$ ethanol and double distilled water at room temperature. All washes were for 1 min under constant agitation. Slides were dried by centrifugation at 1200 r.p.m. for $6 \mathrm{~min}$ before proceeding to hybridisation under LifterSlips (Eerie Scientific, Portsmouth, NH, USA) at $42{ }^{\circ} \mathrm{C}$ overnight in a humid hybridisation chamber.

Post-hybridisation, the slides were washed for $10 \mathrm{~min}$ at room temperature in the following solutions: $2 \times \mathrm{SSC}+$ $0 \cdot 2 \%$ SDS, $2 \times$ SSC and $0.2 \times$ SSC before being spun dry and scanned. They were scanned at 635 (Cy5 Dye) and $532 \mathrm{~nm}$ (Cy3 Dye) in a GenePix 4000B Scanner (Axon Instruments, Foster City, CA, USA). Median fluorescent data were collected for each wavelength for each gene. Fluorescent data were analysed by the statistical software BRB Array Tools (Biometric Research Branch, National Cancer Institute, MD, USA, http://linus.nci.nih.gov/ BRB-ArrayTools.html). Data were normalised by applying a Lowess smoother and genes were excluded if less than $20 \%$ of expression data had at least a $1 \cdot 5$-fold change in either direction from a gene's median value, or if more than $50 \%$ of data were missing or filtered out. Log ratios were calculated comparing the fluorescence data for the reference (at $532 \mathrm{~nm}$ ) with the sample (WT or $\mathrm{KO}$, at $635 \mathrm{~nm}$ ). Class comparison analysis was performed by two sample $t$-tests comparing the WT log ratio with the KO log ratio. An increase or decrease that was statistically significant is expressed as up- $(\uparrow)$ or down- $(\downarrow)$ regulation of a gene in text and tables. Statistical significance was accepted when $P<0 \cdot 05$.

Genes which were significantly changed were analysed by Biorag software (Bio Resource for Array Genes, http://www.biorag.org) and classified based on gene ontology terms.

\section{Quantitative real-time PCR analysis}

Quantitative RT-PCR analysis was performed on selected genes identified as significantly altered in $\mathrm{Npr1-/-}$ versus WT by microarray analysis, in order to quantitate the expression of genes of interest. Genes were selected if several members of a signalling pathway were significantly altered in more than one of the Npr1 - / - versus WTage or gender groups. We used the total RNA samples from the same individuals for both microarray and RT-PCR analyses. The cDNA was generated from $2.5 \mu \mathrm{g}$ ventricular total RNA (after treatment with RNase-free DNase 1; Roche, Mannheim, Germany) for each Nprl WT and Npr1-/- RNA sample using Superscript III Reverse Transcriptase (200 U/ $\mu \mathrm{l}$, Invitrogen). cDNA products were then treated with $1 \mu \mathrm{l}$ Rase $\mathrm{H}(2 \mathrm{U} / \mu \mathrm{l}$, Invitrogen) for $20 \mathrm{~min}$ at $37^{\circ} \mathrm{C}$.

The PCR conditions were optimised for each gene of interest, and the sequences of PCR products were confirmed by sequencing on an ABI 3100-Avant Genetic Analyser (Foster City, CA, USA) before realtime PCR analysis. Levels of mRNA expression were evaluated by quantitative RT-PCR in a Rotor-Gene RG3000 real-time PCR machine (Corbett Research, Sydney, Australia). Oligonucleotide primer sequences and PCR annealing temperatures for each gene studied are given in Table 1. Reactions incorporated the fluorescent dye SYBR Green 1 (Roche), and absolute

Table 1 Primer sequences and annealing temperatures for RT-PCR assays

Primers

\begin{tabular}{|c|c|}
\hline mRNA & \\
\hline ANP & f GAACCTGCTAGACCACCT \\
\hline & r CCTAGTCCACTCTGGGCT \\
\hline BNP & $\begin{array}{l}\text { f AAGCTGCTGGAGCTGATAAGA } \\
\text { r GTTACAGCCCAAACGACTGAC }\end{array}$ \\
\hline Mef2C & $\begin{array}{l}\text { f ATTTGGGAACTGAGCTGTGC } \\
\text { r CGCTCATCCATTATCCTCGT }\end{array}$ \\
\hline Hdac $7 a$ & $\begin{array}{l}\text { f ATCTCTTCCTGGCAGGCTTA } \\
\text { r TTCTGCTTGACCACACTGCT }\end{array}$ \\
\hline $\mathrm{PKCl}$ & $\begin{array}{l}\text { f CTACCGCGGGGATATTATGA } \\
\text { r TCAGCTCGTACAGCCTGAAA }\end{array}$ \\
\hline GATA4 & $\begin{array}{l}\text { f AATGCCTGTGGCCTCTATCA } \\
\text { r CTGGTTTGAATCCCCTCCTT }\end{array}$ \\
\hline Collagen 1 & $\begin{array}{l}\text { f AGGCTTCAGTGGTTTGGATG } \\
\text { r CACCAACAGCACCATCGTTA }\end{array}$ \\
\hline Calmodulin 1 & $\begin{array}{l}\text { f AGGGGTTTGGAGGTGACTTT } \\
\text { r TTCCTCGGAGGTTAGGGTTT }\end{array}$ \\
\hline Phospholamban & $\begin{array}{l}\text { f ATTTCGCCTCCTTACCTCCA } \\
\text { r AGCTTCAGCGTCACGTTTCT }\end{array}$ \\
\hline TGF- $\beta 1$ & $\begin{array}{l}\text { f TGAGTGGCTGTCTTTTGACG } \\
\text { r GGTTCATGTCATGGATGGTG }\end{array}$ \\
\hline
\end{tabular}

Annealing temperature $\left({ }^{\circ} \mathrm{C}\right)$

56

56

56

54

54

58

54

54

54

54

Sequences are listed $5^{\prime}-3^{\prime}$. Forward primers are designated by $f$ and reverse primers by $r$. 
gene expression levels were calculated by generating individual standard curves for each gene as described by Karsai et al. (2002). Standard curves comprising at least six points were run with each assay, with concentrations ranging from 0 to $2000 \mathrm{pg}$, with each concentration run in triplicate. Hotmaster Taq DNA Polymerase (Eppendorf, Hamburg, Germany) was used in all reactions. For each assay, a hotstart at $96{ }^{\circ} \mathrm{C}$ for 2 min was performed before the following PCR cycling parameters: denaturation at $94{ }^{\circ} \mathrm{C}$ for $30 \mathrm{~s}$, annealing for $35 \mathrm{~s}$ at the gene-specific annealing temperature (Table 1) and extension at $72{ }^{\circ} \mathrm{C}$ for $30 \mathrm{~s}$. Each sample underwent 30 cycles, after which a melt curve was performed. Each sample was assayed in duplicate and gene levels expressed as picograms of message per microgram of total RNA (pg/ $\mu \mathrm{g}$ total RNA). Fluorescent data were acquired at each extension step.

\section{Cardiac function}

Cardiac function experiments were performed on 8-week- and 6-month-old male WT, heterozygous and Npr1-/- mice. The isolated perfused mouse heart preparation was similar to that described previously (Eklund et al. 2001). Briefly, mice were anaesthetised with sodium pentobarbitone $(50 \mathrm{mg} / \mathrm{kg}$, i.p.), decapitated and the hearts rapidly cooled with ice-cold buffer, prior to retrograde perfusion by the Langendorff technique. The flow rate was adjusted according to the weight and genotype of the animal to achieve a similar perfusion per gram of tissue. To measure the isometric force of contraction, the left atrium was cut off and an empty plastic balloon was inserted into the left ventricle. The balloon was filled with $50 \%$ ethanol to give an end-diastolic pressure of approximately $5 \mathrm{mmHg}$, and the developed pressure inside the balloon was recorded with a pressure transducer (Capto, model SP844). A similar pressure transducer was connected to a sidearm cannula to monitor the aortic perfusion pressure. The hearts were paced with a stimulator (Digitimer Ltd, Hertfordshire, UK; 400 beats per minute at baseline), and all recordings were made by an ADInstruments Powerlab system. The comparison of contractile function was done at corresponding levels of end-diastolic pressure because of the difference in the cardiac size between the experimental groups. The data were obtained during the stepwise filling of the left ventricular balloon as described previously (Stromer et al. 1997, Piuhola et al. 2003).

\section{ANP secretion}

The cardiac secretion of ANP was analysed from coronary effluents from isolated hearts from 8-weekand 6-month-old male mice. At baseline, the 8-week hearts were perfused with a coronary flow rate of $21 \cdot 7 \pm$ 1.4 and $20.5 \pm 1.1 \mathrm{ml} / \mathrm{g}$ cardiac weight, for WT and Npr1-/ - mice respectively, resulting in a coronary perfusion pressure of $83 \pm 5$ and $91 \pm 10 \mathrm{mmHg}$. For the RIA of ANP, timed collection of coronary effluent was obtained, the samples were cooled on ice and stored at $-20{ }^{\circ} \mathrm{C}$ until SepPak extraction and RIA was performed as described by Yandle et al. (1991).

\section{Statistical analysis}

All results (except microarray data) are expressed as means \pm s.E.M. The effects of WT versus $\mathrm{Nprl}-/-$, age and gender on cardiac gene expression data were tested by three-way factorial ANOVA. Subsequent comparisons between WT and Npr1 - / - of specific age and gender groups were conducted using independent $t$-tests. The effects of genotype on cardiac ANP secretion and developed pressure were tested by one-way ANOVA. Associations between variables were tested using Pearson's correlation coefficient. Values of $P<0 \cdot 05$ were considered statistically significant.

\section{Results}

\section{Hypertension and cardiac hypertrophy}

Mean arterial pressure (MAP) was significantly increased over all Npr1 - / - groups when compared with WT (Fig. 1A, $P<0 \cdot 001$ ), equally at 8 weeks and 6 months. On an average, there was a $32 \mathrm{mmHg}$ difference in MAP between Npr1 - / - and WT groups, and there were no significant differences between blood pressures dependent on age or gender. In contrast, heart weight to body weight ratios (HW:BW) of Npr1-/- mice increased significantly in all groups when compared with WT by ANOVA $(P<0 \cdot 01$, Fig. 1A). The most pronounced increase was observed in male Npr1-/mice at 8 weeks of age. In 8 -week-old male mice, HW:BW was $169 \%$ when compared with WT $(6 \cdot 6 \pm 0.5$ vs $3 \cdot 9 \pm$ $0 \cdot 3)$. However, the relative hypertrophy observed in young adult male Npr1-/ - mice was not sustained, and at 6 months the HW:BW of Npr1 $-/-$ male mice was only $118 \%$ relative to WT $(6 \cdot 9 \pm 0 \cdot 5$ vs $5 \cdot 8 \pm 0 \cdot 1)$. In contrast, in female Npr1 $-/-$ mice, the proportionate increase in HW:BW was similar at both 8 weeks and 6 months of age. The HW:BW of female Npr1-/- mice at 8 weeks of age was $135 \%$ when compared with WT $(5 \cdot 3 \pm 0 \cdot 2$ vs $3 \cdot 9 \pm 0 \cdot 3)$, and at 6 months of age was $134 \%$ when compared with WT $(6 \cdot 76 \pm 0 \cdot 4$ vs $5 \cdot 04 \pm 0 \cdot 3)$. These results suggest that maximal hypertrophy occurred at an early age in males, whereas in females the hypertrophic response was sustained as the female mice aged. 

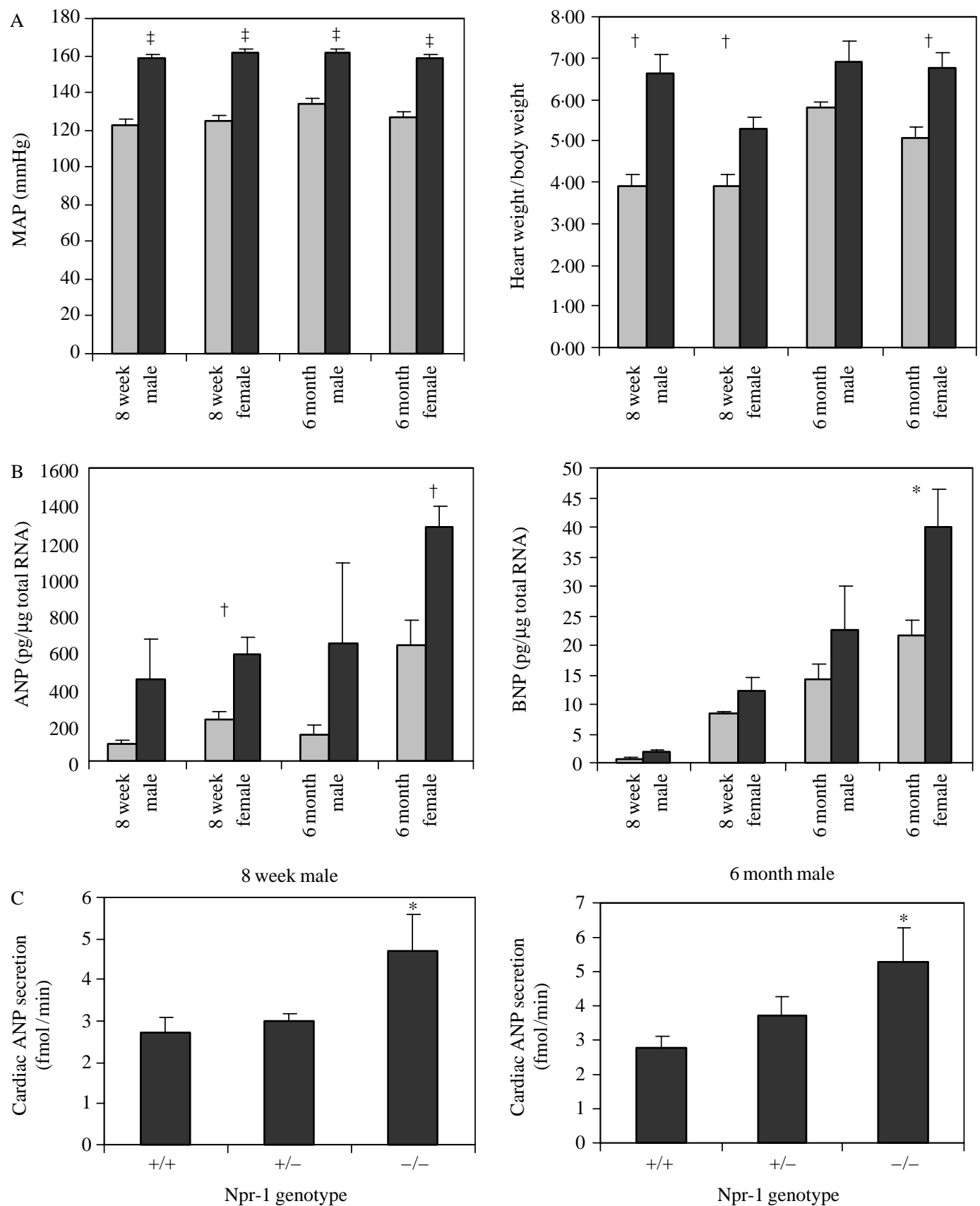

Figure $1 \mathrm{MAP}$ and $\mathrm{HW}: \mathrm{BW}$ ratios $(\mathrm{A})$, ventricular levels of ANP and BNP mRNAs $(\mathrm{B})$ in male and female Npr1-/- mice at 8 weeks and 6 months of age when compared with WT control mice (quantitated gene levels are expressed as picograms of message per microgram of total RNA), and ANP secretion rates from isolated perfused hearts of $K O(-/-)$, heterozygote $(-/+)$ and WT $(+/+)$ Npr1 male mice at 8 weeks and 6 months of age $(C)$. Black bars represent Npr1 KO and grey bars represent WT $(A$ and B). Significant differences between WT and $\mathrm{KO}$ at individual age/gender points are indicated by ${ }^{*} P<0.05,{ }^{\dagger} P<0.01$ and ${ }^{\ddagger} P<0.001$.

\section{Microarray analysis of cardiac gene expression}

To investigate the pathways regulated by Npr1 in the development of cardiac hypertrophy, cDNA microarray analysis was used as a screening tool to indicate genes with altered expression in the ventricles of male and female Npr1 - / - mice at 8 weeks and 6 months. Out of a total of 22656 genes represented on the Compugen microarray slides, 187 were significantly changed $(P<0 \cdot 05)$ between 8-week male WT and Npr1-/-, 
when compared with 248 genes in 8-week females. In contrast, at 6 months, 284 genes were significantly changed between WT and Npr1-/- males, when compared with 567 genes in 6-month female mice ( $n=6$ per group). The microarray data of hypertrophyrelated genes that were significantly altered are shown in Table 2. The most significantly altered genes include ANP, calmodulin and calmodulin kinase, protein kinases and MAP kinases, and several transcription factors, notably members of the Hdac, Mef2, NFAT and GATA families. In addition, altered cell-signalling pathways involved in fibrosis were observed, including fibroblast growth factor, collagen and matrix metalloproteinases. This led us to focus the real-time PCR studies on key genes involved in these pathways. Other genes whose expression was significantly altered in the microarray data but have not been studied further in the present study included genes involved in cardiac development (including forkhead box proteins and catenin), receptor activity (guanine nucleotide binding protein) and the regulation of transcription (nuclear factor interleukin-3, insulinoma-associated 2, zinc-finger proteins, general transcription factors, ankyrins). In addition, genes involved in heart-rate regulation (nicotinic cholinergic receptor) and ion channel signalling (ryanodine receptor, solute carrier family, voltage-gated sodium channels and $\mathrm{K}^{+}$channels) were also significantly altered but not examined further in this study. Genes involved in the regulation of transcription were particularly increased at 8 weeks of age in male Npr1-/-; however, in female animals, up-regulation of transcription factor genes became more apparent at 6 months of age. These gender differences in the age at which transcription factors were elevated, paralleled the times when their maximal HW:BW ratio occurred (also at 8 weeks in male and 6 months in female knockouts). Intriguingly, gene ontology classification indicated that the largest proportion of genes with altered expression is those whose function is presently unknown.

\section{Quantitative real-time PCR analysis}

Quantitative real-time PCR analysis was used to characterise expression levels in genes of interest selected after collating the microarray data from all groups. As expected, ventricular ANP gene expression was significantly increased in $\mathrm{KO}$ mice over all age and gender groups $(P=0 \cdot 002)$, particularly in female Npr1-/mice at both 8 weeks and 6 months (Fig. 1B). Ventricular BNP expression was also greater over all Npr1-/groups compared with WT mice when compared by three-way factorial ANOVA $(P=0 \cdot 005)$. Levels of BNP mRNA significantly increased with age $(P<0 \cdot 001)$ and females had higher levels than males at both the ages $(P<0 \cdot 001)$. BNP mRNA was particularly increased in 6 -month female Npr1 $-/-$ mice, as indicated by $t$-test analysis $(P<0 \cdot 05)$.
In 8-week-old males, the average ANP secretion rate per minute was $4.7 \pm 0.9$ and $2 \cdot 7 \pm 0.4 \mathrm{fmol} / \mathrm{min}$, for Npr $1-/-$ and WT mice respectively $(P<0 \cdot 05, n=5$ for both the genotypes). Similar results were seen in the 6 -month-old mice, with ANP secretion levels of $5 \cdot 3 \pm 1$ and $2 \cdot 8 \pm 0.3 \mathrm{fmol} / \mathrm{min}(P<0 \cdot 05)$, for $\mathrm{Nprl}-/-$ and WT respectively (Fig. $1 \mathrm{C}$ ).

A gene that is central to hypertrophic signalling is calmodulin. Gene expression of calmodulin 1 was significantly increased in Npr1-/ - when compared with WT overall $(P=0 \cdot 013)$, and especially in 8-week females (Fig. 2A). It should be noted, however, that the most significant change in calmodulin 1 was the remarkable increase in expression with age in both the genotypes $(P<0 \cdot 001)$. Ventricular $P K C$ gene expression showed an interaction with genotype, age and gender $(P=0 \cdot 008)$, whereby expression was significantly increased in female Npr1-/ - when compared with $\mathrm{WT}$ at 6 months, but tended to be decreased in 6-month Npr1 - / - males (Fig. 2B). Gene expression of $\mathrm{Hdac} 7 \mathrm{a}$ was increased in $\mathrm{Npr} 1-/-$ males versus WTat both 8 weeks and at 6 months $(P<0 \cdot 05)$. No change in Hdac 7 a expression was seen in females at either age (Fig. 2E). Analysis of ventricular expression of the transcription factor, Mef2C, by RT-PCR did not show significant differences in Nprl-/- when compared with WT mice at either age (Fig. 2C), in contrast to the microarray data. However, Mef2C expression was markedly increased with age in both the genotypes $(P<0 \cdot 001)$. Ventricular expression of GATA4 was significantly increased with genotype in the Npr1 - / mice $(P=0.03)$, particularly in 6 -month female Npr1 $-/-$ animals (Fig. 2D). In addition, there was a significant increase in GATA4 with age $(P<0 \cdot 001)$.

Among genes involved in fibrosis, collagen 1 expression was increased in Nprl $-/-$ mice of both the genders $(P=0 \cdot 011)$, although this was particularly evident in 6-month female Npr1-/- mice (Fig. 3A). Ventricular expression of phospholamban and transforming growth factor- $\beta 1$ (TGF- $\beta 1$ ) were the only genes which significantly decreased with age $(P<0 \cdot 001)$. TGF$\beta 1$ gene expression was greater in Npr1 - / - versus WT males at 6 months (Fig. 3B). Phospholamban was significantly decreased in 6-month female Npr1-/versus WT (Fig. 3C).

\section{Gene correlations}

The majority of hypertrophy-related genes were strongly correlated with HW:BW (except collagen 1 and TGF- $\beta 1$ ), and with each other, including ANP, BNP, Mef2c, Hdac 7a, protein kinase C (PKC) ı, GATA4 and calmodulin $1 \quad(r=0 \cdot 322-0 \cdot 948)$. Collagen 1 expression was significantly correlated with $\mathrm{HW}: \mathrm{BW}$ and MAP, but was not correlated with any of the hypertrophy-related genes. While MAP was 
Table 2 Microarray analysis data showing hypertrophy related genes significantly altered in Npr1-/- versus WT groups $(P<0 \cdot 05)$

$\mathrm{Ca} / \mathrm{Calmodulin}$

Calmodulin kinase (NM_012040)

Calmodulin 1 (NM_009790)

Ion transport

Ryanodine receptor 1 (X83932)

Solute carrier family 22, member 4 (NM_019687)

Chloride ion channel inducer protein (U53455)

Solute carrier family 4 (anion exchanger) (NM_011403)

$\mathrm{K}^{+}$channel subfamily U1 (NM_008432)

$\mathrm{Na}^{+} / \mathrm{H}^{+}$exhanger (AF139195)

$\mathrm{K}^{+}$channel protein (AJ291608)

Maxi $\mathrm{K}^{+}$channel- $\beta$ subunit (AF020711)

Voltage gated sodium channel (L36179)

Voltage gated sodium channel type 1- $\beta$

Solute carrier family 22, member 21 (NM_019723)

Cell signalling

Mitogen-activated protein kinase 7 (MAPK7) (NM_011841)

Protein kinase C, iota (NM_008857)

Protein kinase inhibitor, $\gamma$ (NM_011106)

Mitogen-activated protein kinase 4 (NM_008696)

Mitogen-activated protein kinase kinase kinase 14 (Map3k14;

NM 016896)

Guanine nucleotide-binding protein $\gamma$-11 (NM_025331)

Mek kinase 3 (U43187)

Mitogen-activated protein kinase kinase kinase kinase 3 (BC006037)

Protein kinase C, $\zeta$ (NM_008860)

Mitogen-activated protein kinase 3 (Erk-1; Z14249)

Transcription factors

Myocyte-specific enhancer factor 2 (Mef2C; L13171)

Insulinoma-associated 2 (NM_020287)

Nuclear factor, interleukin-3 regulated (NM_017373)

Transcription factor 3 (NM_009332)

Forkhead box N1 (NM_008238)

Kruppel-like factor 12 (NM_010636)

Histone deacetylase 7A (NM_019572)

Histone deacetylase 8 (AK011332)

General transcription factor II A,1 (NM_031391)

CCAAT/enhancer-binding protein (NM_009883)

Zinc-finger protein 57 (NM 009559)

Forkhead box G1 (NM_008241)

Forkhead box N4 (AF323488)

Catenin $\beta$ (NM_007614)

Zinc-finger homeobox 1a (NM_011546)

General transcription factor III C1 (BC004708)

Transcription factor AP-2 $\alpha$ (NM 011547)

Ankyrin repeat domain 1 (NM_013468)

Ankyrin repeat domain $2 \mathrm{~S}$ (BC006647)

Homeobox B13 (NM_008267)

Transcription factor 19 (BC004617)

TAF7 RNA polymerase II, TATA box-binding factor (NM_011901)

Fibrosis

Fibroblast growth factor 3 (NM_008010)

Matrix metalloproteinase 15 (NM_008609)

Matrix metalloproteinase 7 (NM 010810 )

Matrix metalloproteinase 3 (NM_010809)

Fibroblast growth factor inducible 15 (NM 008016)

Fibroblast growth factor homologous factor 3 isoform 1B (AF199604)

Matrix metalloproteinase 8 (NM_008611)

Fibroblast growth factor 3 (NM_008007)

Other

Procollagen type $8 \alpha-1$ (NM_007739)

Atrial natriuretic peptide (K02781)

Purinergic receptor P2X, ligand gated ion channel 5 (NM_033321)

Cholinergic receptor, nicotinic, $\beta 2$ (NM_009602)

Cholinergic receptor, nicotinic, $\alpha$-polypeptide1 (NM_007389)

Glyceraldehyde-3-phosphate dehydrogenase (NM_008084) $\uparrow$ 6-Month males

$\uparrow 6$-Month females

$\downarrow$ 8-Week females, $\downarrow$ 6-month males

$\downarrow$ 8-Week females

$\downarrow$ 8-Week females

$\downarrow$ 6-Month males

$\downarrow$ 6-Month males

$\uparrow$ 6-Month males

$\downarrow$ 6-Month males

$\downarrow$ 6-Month males

$\downarrow$ 6-Month females

$\uparrow$ 6-Month females

$\downarrow$ 6-Month females

$\uparrow$ 8-Week males

$\uparrow$ 8-Week males

$\downarrow$ 8-Week males

$\downarrow$ 8-Week females

$\downarrow$ 8-Week females

$\uparrow$ 6-Month males

$\downarrow$ 6-Month females

$\downarrow$ 6-Month females

$\uparrow 6$-Month females

$\downarrow$ 6-Month females

$\uparrow 8$-Week males

$\uparrow$ 8-Week males

$\uparrow$ 8-Week males

$\uparrow$ 8-Week males

$\uparrow$ 8-Week males, $\downarrow$ 6-month males

8-Week males

$\downarrow$ 8-Week females

$\downarrow$ 8-Week females

8-Week females

$\downarrow$ 8-Week females

$\downarrow$ 8-Week females

$\uparrow$ 6-Month males

6-Month males

$\uparrow 6$-Month males

$\uparrow 6$-Month males

$\downarrow$ 6-Month males

6-Month males

6-Month females

$\uparrow$ 6-Month females

$\downarrow$ 6-Month females

$\downarrow$ 6-Month females

$\uparrow 6$-Month females

$\uparrow$ 8-Week males

$\downarrow$ 8-Week males

$\downarrow$ 8-Week males

$\uparrow$ 8-Week males

$\downarrow$ 8-Week females

8-Week females

6-Month females

$\downarrow$ 6-Month females

$\uparrow$ 6-Month females

$\uparrow$ 8-Week males, $\uparrow$ 8-week females

$\downarrow$ 8-Week males

$\downarrow$ 8-Week females

$\uparrow$ 6-Month females

$\uparrow$ 8-Week males

GenBank accession numbers are shown in brackets. 

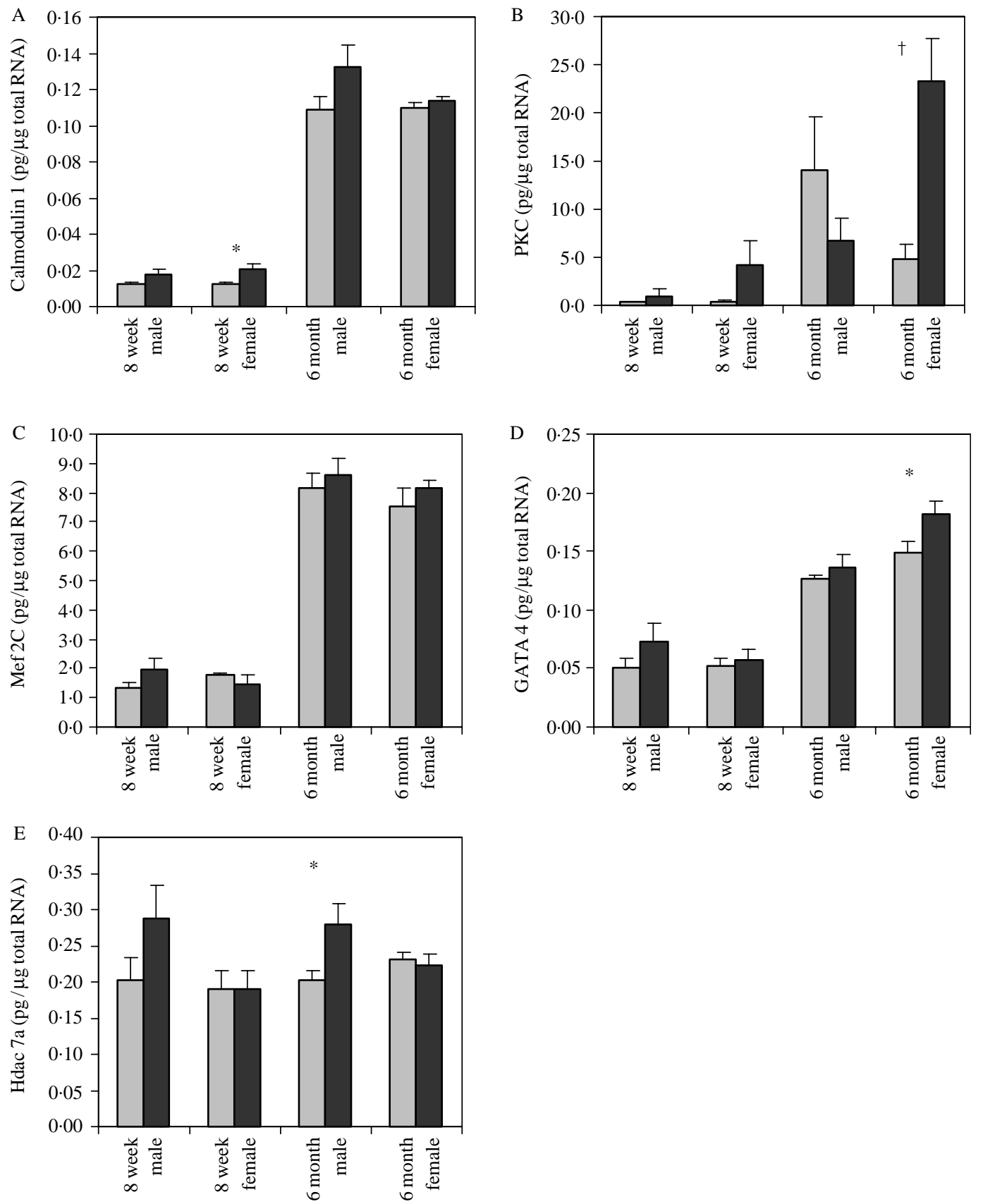

Figure 2 Ventricular levels of calmodulin 1 (A), PKCl (B), Mef2C (C), GATA4 (D) and Hdac 7a (E) mRNAs in male and female Npr1-/mice at 8 weeks and 6 months of age when compared with WT control mice. Quantitated gene levels are expressed as picograms of message per microgram of total RNA (pg/ $\mu \mathrm{g}$ total RNA). Black bars represent Npr1 KO and grey bars represent WT. Significant differences between WT and KO mice are indicated by ${ }^{\star} P<0.05$ and ${ }^{\dagger} P<0.01$.

significantly correlated with ANP, BNP and PKC, it was not correlated with other hypertrophic or fibrotic genes, in agreement with previous reports that these pathways are independent of the blood pressure elevation in the Npr1-/- mice.

\section{Cardiac function}

\section{Cardiac contractility and response to stress}

The cardiac contractility of WT and Npr1-/- male mouse hearts was compared at different levels of 

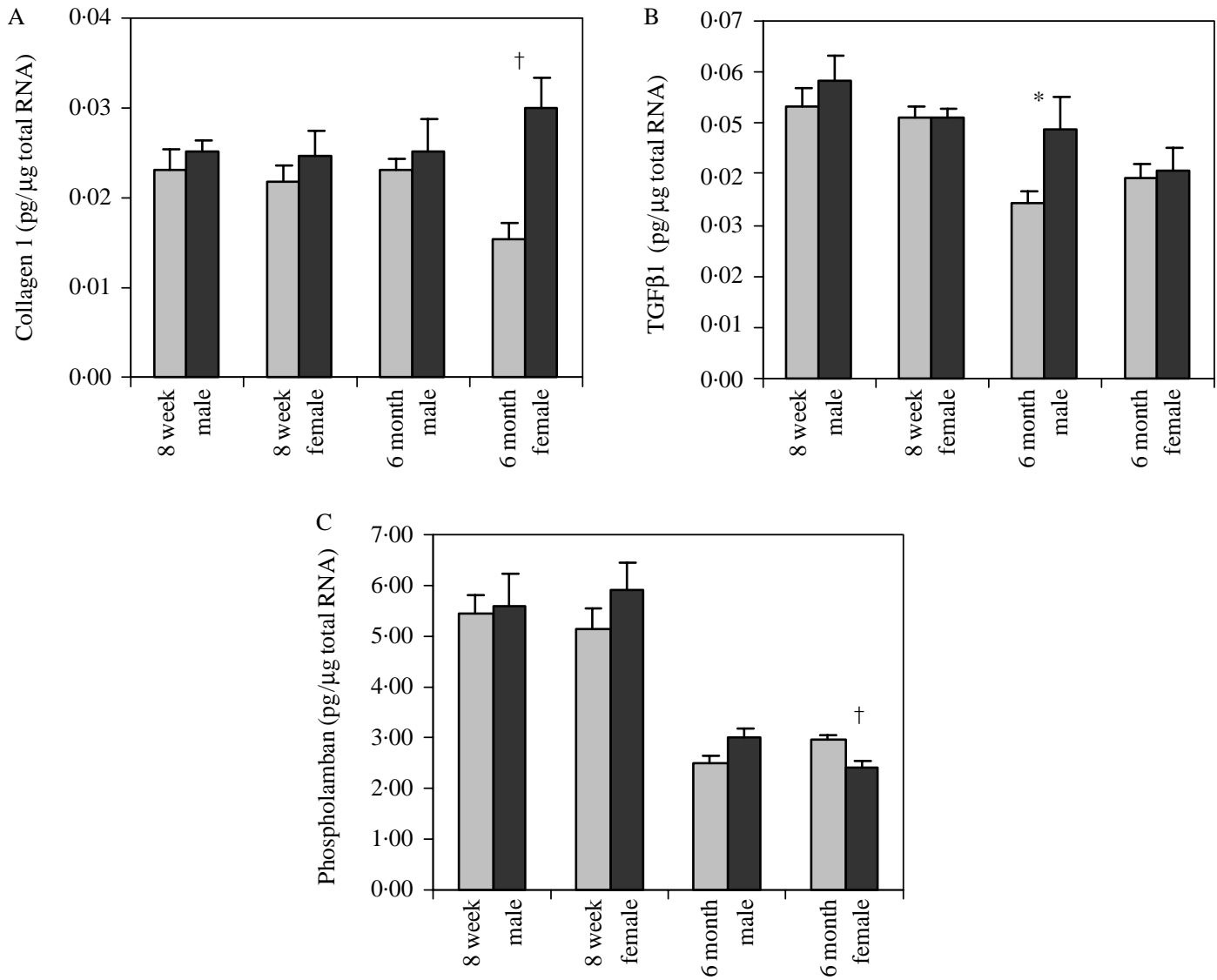

Figure 3 Ventricular levels of collagen 1 (A), TGF $\beta-1$ (B) and phospholamban (C) mRNAs in male and female Npr1-/- mice at 8 weeks and 6 months of age when compared with WT control mice. Quantitated gene levels are expressed as picograms of message per microgram of total RNA (pg/ $\mu$ g total RNA). Black bars represent Npr1 KO and grey bars represent WT. Significant differences between WT and KO mice are indicated by ${ }^{*} P<0.05$ and ${ }^{\dagger} P<0.01$.

end-diastolic pressure in isolated Lagendorff-perfused hearts. Throughout the pressure range used, the hearts of 8-week-old Npr1-/- mice showed enhanced contractility when compared with those from WT animals (Fig. 4). In 8-week-old mice, the developed pressure (DP) was $18.7 \pm 1.8 \mathrm{mmHg}$ in WT and $27 \cdot 2 \pm$ $2 \cdot 2 \mathrm{mmHg}$ in Npr $1-/-$ mice at the left ventricular enddiastolic pressure (LVEDP) of $10 \mathrm{mmHg}(P<0 \cdot 005)$. In 6 -month-old mice, this contractility enhancement was lost, and there was no difference in the contractility between the different genotypes (DP $33.5 \pm 3.9 \mathrm{mmHg}$ in WT and $34 \cdot 0 \pm 4.8 \mathrm{mmHg}$ in $\mathrm{Npr} 1-/-$; at LVEDP, $10 \mathrm{mmHg}$; $P$, not significant).

\section{Discussion}

The present study implicates the calmodulin-CaMKHdac-Mef2 and PKC-MAPK-GATA4 pathways in Npr1 mediation of cardiac hypertrophy, extending our previous understanding of Nprl involvement in the AT1R-calcineurin-NFAT pathway. Increased expression of genes of the $\mathrm{Ca}$ /calmodulin pathways was observed early in the development of cardiac remodelling in Npr1-/ - mice, whilst other genes involved in cardiac hypertrophic-signalling pathways including $\mathrm{PKCl}$, Hdac $7 \mathrm{a}$ and GATA4 tended to be increased in Npr1 - / - mice at a more advanced stage. Structural molecules involved in cardiac fibrosis such as collagen 1 also tended to be increased later in the remodelling process.

One of the startling observations of this study was the age-related change in gene expression, independent of genotype. In particular, the expression of cardiac transcription factors Mef2C and GATA4, as well as calmodulin 1, were markedly increased at 6 months when compared with 8 weeks in both knockout and wildtype mice. Over the same period, there were significant decreases in expression levels of phospholamban and 

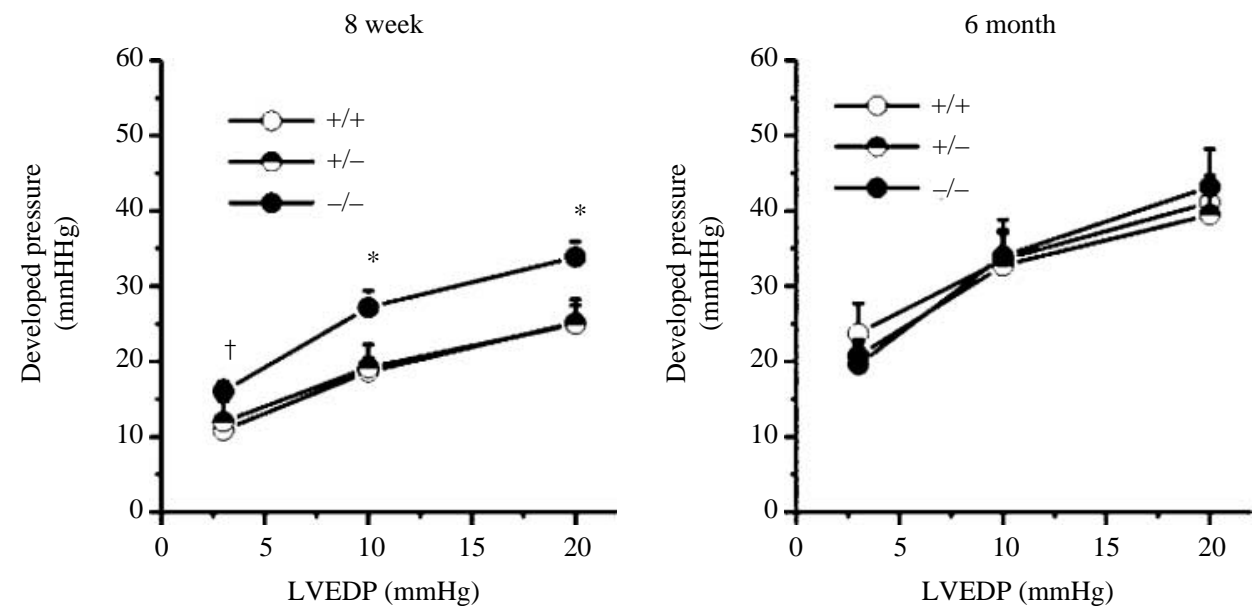

Figure 4 The contractile function at different levels of left ventricular end-diastolic pressure (LVEDP) in WT $(+/+)$, heterozygous $(-/+)$ and $\mathrm{KO}(-/-)$ Npr1 male mice at 8 weeks and 6 months of age. At 8 weeks, the KO Npr1 mice had significantly enhanced contractility throughout the range of developed pressures $\left({ }^{*} P<0.05,{ }^{\dagger} P<0.01, \mathrm{Npr} 1 \mathrm{KO}\right.$ versus WT). At 6 months, this enhancement of contractility was lost and there were no differences in cardiac function among genotypes.

TGF- $\beta 1$. To our knowledge, there have been no other reports in the literature of marked changes in gene expression associated with normal cardiac aging in the absence of pathophysiological changes. However, the phenotypic plasticity of adult myocardium is well documented, associated with the reactivation of the foetal gene programme and the expression of at least four transcription factors, GATA4, MEF2, Csx/Nkx2-5 and e/dHAND (Swynghedauw 2006). This activation is normally attributed to cardiac mechanical overload and wall stress, acting via stretch-activated $\mathrm{Ca}, \mathrm{K}$ and $\mathrm{Na}$ channels and calcineurin (Swynghedauw 2006). It may be that arterial stiffness in the aging cardiovascular system leads to increased arterial pre-load, wall stress in the heart and the gradual activation of cardiac transcription. These findings highlight the necessity that gene expression studies are performed on precisely age-matched animals. In the present study, however, significantly altered gene expression was demonstrated in Npr1-/- mice when compared with age-matched wild-type animals, indicative of cardiac remodelling over and above the age-related changes.

To determine whether the cardiac remodelling observed in aging Nprl $-/-$ mice influences cardiac function, the contractile response to elevated ventricular stretch was studied in perfused isolated hearts. Throughout the pressure range used, 8-week-old male Npr1-/- mice showed enhanced contractility when compared with the wild-type animals. In 6-month-old animals, this enhancement of contractility was lost. These results suggest that in 8-week-old Npr1-/mice, the left ventricular hypertrophy enhances contractile force, whereas older Npr1-/- mice show impaired contractile function, possibly as a result of the increasing collagen deposition and developing heart failure. Enhancement of cardiac contractility has been reported in other models of left ventricular hypertrophy in rats (Stromer et al. 1997, Piuhola et al. 2003). Consistent with the present study, in another strain of Npr1-/- mice, Kuhn et al. (2002) also reported impaired cardiac function in older mice (12 months old), but no differences in cardiac function between mouse genotypes at 4 months of age.

Taken together, the microarray analysis revealed marked differences between genders in gene expression patterns, with male Npr1-/- mouse hearts demonstrating a greater activation of hypertrophy signals in early adulthood when compared with female Npr1-/- animals. Female Npr1-/- mice exhibited their greatest increase in HW:BW and gene expression of ANP, BNP, PKC-MAPK and GATA4 at 6 months, suggesting a more delayed but sustained hypertrophic response in female mice. Many previous studies have established that male mice are more sensitive than female to various genetic interventions leading to cardiac hypertrophy, as has been comprehensively summarised by Du (2004). These findings are in keeping with the observations from other species, including humans, that males in general have an earlier transition into heart failure than females. However, the mechanism underlying sex differences in cardiovascular risk is poorly understood. Although deleterious effects of androgens and beneficial effects of ovarian hormones have been proposed, recent studies suggest that male hormones have a neutral or beneficial effect on the heart (Muller et al. 2003). A previous study of Npr1-/ - mice proposed a role for testosterone in aggravating the hypertrophy in young, male mice ( $\mathrm{Li}$ et al. 2004), in which the castration of Npr1 KO male mice reduced cardiac hypertrophy and fibrosis, whereas 
testosterone infusion in ovariectomised female Npr1-/ - mice increased cardiac mass and fibrosis. The castration experiments in the study demonstrated that androgens accounted for most of the gender differences in hypertrophy and $50 \%$ of the genderrelated cardiac fibrosis. Furthermore, Li et al. (2004) showed that gender differences in $\mathrm{Nprl}-/-$ mice were almost abolished by deleting the angiotensin II type $1 \mathrm{~A}$ receptor (AT1A), suggesting that androgens contribute to gender-related differences in cardiac remodelling via an interaction of Nprl and AT1A receptors. These authors suggested that this may involve TGF- $\beta 1$ and TGF- $\beta 3$, which are activated by AT1A and are responsible for interstitial fibrosis. In the present study, a significant increase in TGF- $\beta 1$ expression was observed in 6-month-old male knockouts, consistent with the influence of testosterone on this pathway. Other molecular pathways proposed to mediate the gender difference in cardiac remodelling include tissue hormones and transcription factors, including Akt and Mef2 and ion channels, particularly calcium channels (Du 2004).

Calmodulin is a key signalling messenger mediating the actions of calcium (Frey et al. 2000), and is a central mediator of several hypertrophic-signalling pathways (Fig. 5). The calcium/calmodulin-dependent enzymes, calcium/calmodulin-dependent protein kinase
(CaMK) and phosphatase calcineurin, have been implicated in the control of cardiac hypertrophy and failure. In this study, calmodulin 1 was shown by RT-PCR to be increased over all Npr1-/- groups. These findings suggest that calmodulin may be a key signal in the cardiac hypertrophy that occurs in response to the lack of Npr1 bioactivity.

One important signal transduction cascade regulated by calmodulin in hypertrophy involves PKC and MAPK. Whilst PKCi is not a classically expressed isoform in cardiac tissue, microarray analysis indicated that this isoform was increased in Npr1-/- mice, and this was confirmed by RT-PCR. Previous studies suggest that PKCt is involved in myocardial ischaemia (Albert \& Ford 1998) and plays a role in actin cytoskeleton organisation (Spitaler et al. 2000). PKC is hypothesised to modulate cardiac hypertrophy by phosphorylation of transcription factors, including Mef2 and NFAT that regulate transcription of hypertrophic genes.

Histone deacetylases are also regulated by calmodulin and calmodulin kinase, and are negative regulators of cardiac hypertrophy that repress activity of the transcription factor Mef2 (Zhang et al. 2002). These chromatin-modifying enzymes have important roles in the control of cardiac hypertrophy (Zhang et al. 2002, Gusterson et al. 2003). In the present study, Hdac 7A and 8 were indicated by both microarray and RT-PCR to

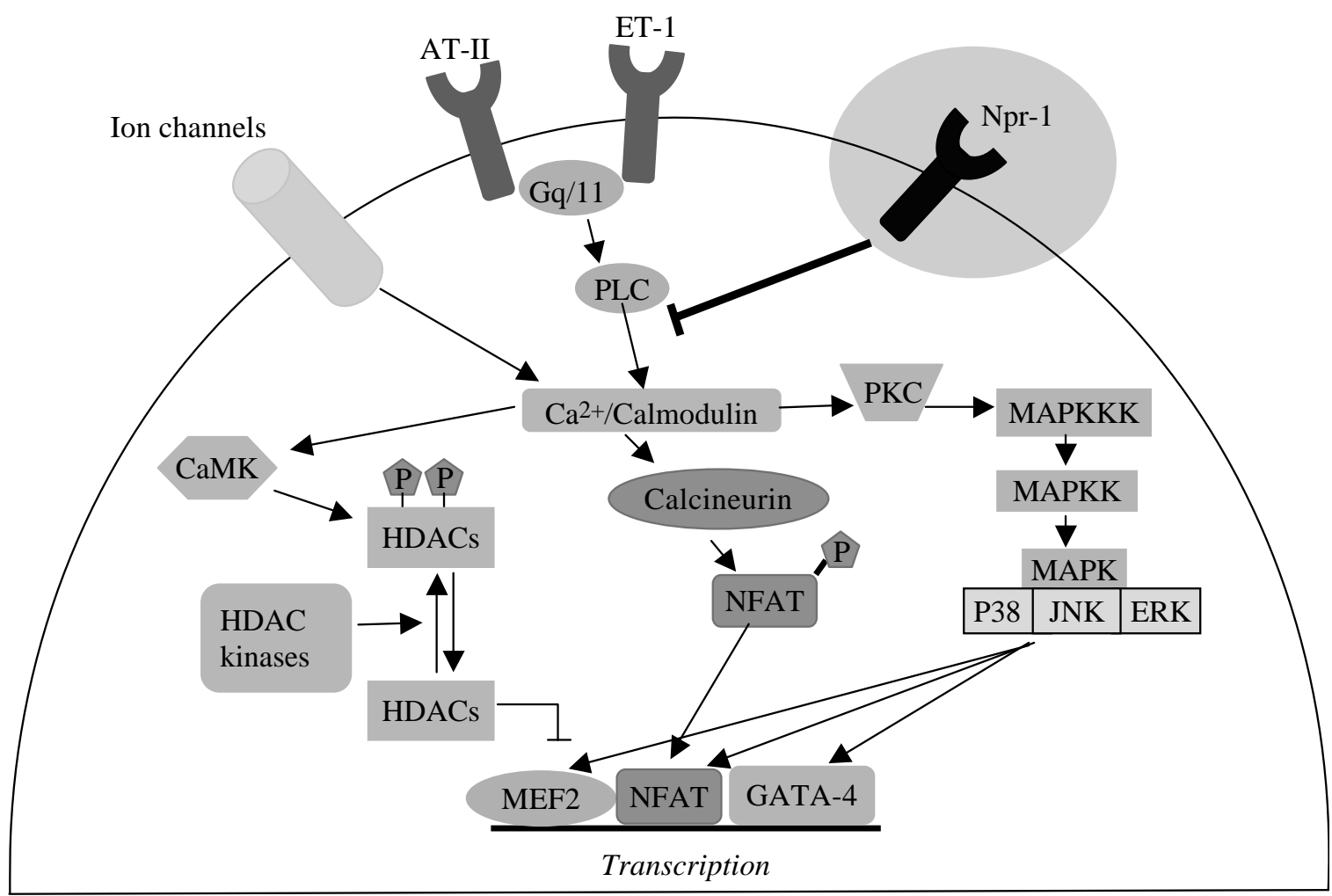

Figure 5 Proposed interaction of Npr1 signalling with pathways contributing to cardiac hypertrophy. 
have sexually dimorphic effects in male and female Npr1 - / - mice, suggesting that Hdacs have a complex role in the development of cardiac hypertrophy in combination with gender, as reported for other genetic mouse models (Du 2004).

GATA4 is another key regulator of inducible cardiac gene expression and a potential mediator of cardiac hypertrophy. The MAP kinases p38 and ERK1/2 stimulate GATA4 activity (Tenhunen et al. 2004), and this transcriptional activity is regulated through physical interaction with NFAT, Mef2 and serum response factor (Adazawa \& Komuro 2003). GATA4 is responsible for regulating the basal expression of cardiac hypertrophy genes including $\alpha$-myosin heavy chain, myosin light chain 1/3, cardiac troponin C and I, ANP, BNP and the sodium-calcium exchanger in cardiac tissue (Adazawa \& Komuro 2003). In our studies, increased GATA4 gene expression was observed in all $\mathrm{Npr} 1-/-$ mice especially in 6-month-old Npr1 KO females. Consistent with this, increased levels of both ANP and BNP mRNA expression in the Npr1-/- ventricle were most marked in 6-month-old females. The reactivation of ANP expression in adult ventricular myocardium has become one of the most sensitive markers of hypertrophy (Day et al. 1987). This increased expression was reflected in increased ANP hormone secretion.

Cardiac fibrosis is a classical feature of hypertrophy and is characterised by the expansion of the extracellular matrix due to the accumulation of collagen, particularly collagen types I and III (Manabe et al. 2002). Such stiffening impedes both the contraction and the relaxation of cardiomyocytes, impairs electrical coupling and can lead to myocyte hypoxia due to reduced capillary density and increased oxygen diffusion distance (Manabe et al. 2002). In the present study, collagen 1 gene expression was increased in both male and female 6-month Npr1-/- animals, but was not apparent in younger mice. TGF- $\beta$ promotes the proliferation of fibroblasts, stimulates extracellular matrix protein production while inhibiting its degradation by induction of antiproteinases or reduction of metalloproteases (Manabe et al. 2002). Transgenic mice overexpressing TGF- $\beta 1$ develop cardiac hypertrophy and interstitial fibrosis (Rosenkranz et al. 2002). The late increase in the expression of collagen 1 and TGF- $\beta$ in 6 -month-old Npr $1-/-$ mice implies that in this mouse model of cardiac disease, hypertrophy occurs in early adulthood, whilst the fibrotic response occurs later as the animals age, concomitant with the decline in cardiac contractility we observed in Npr1-/ - hearts between 8 weeks and 6 months. Finally, a decrease in the expression of phospholamban, a molecule responsible for mediating cardiac relaxation, was observed in 6-month female Npr1-/- animals. Phospholamban mutations have been shown to cause dilated cardiomyopathy and resultant heart failure, but this pathway has not previously been associated with Npr1 activity.

In summary, we have shown that Nprl signalling influences the very earliest steps in the cellular pathways leading to cardiac hypertrophy and fibrosis by first altering the levels of $\mathrm{Ca} /$ calmodulin signalling. This, in turn may lead to observed alterations in the expression of the multiple intracellular messengers, including Hdac's, PKCi and the transcription factor GATA4. Male mice demonstrated an earlier onset of cardiac remodelling, consistent with the onset of human heart disease at a younger age in men when compared with women. We also report marked age-related changes in the levels of expression of several transcription factor genes, independent of genotype, which has implications for the phenotypic plasticity of adult myocardium even in the absence of overt cardiovascular disease. The microarray studies have raised intriguing questions about gene pathways not previously identified with Npr1 signalling. Approximately one-third of genes identified as significantly altered by microarray are of unknown function. Characterisation of these gene pathways will be the subject of ongoing studies.

\section{Acknowledgements}

The authors thank Howard Potter (Molecular Pathology, Canterbury Health Labs) for work that involved sequencing PCR products. This work was supported by the National Heart Foundation of New Zealand, The Heath Research Council of New Zealand and the New Zealand Lotteries Grants Board. Jarkko Piuhola was supported by grants from Sigrid Jusélius Foundation, Finnish Heart Research Foundation, Paavo Nurmi Foundation and Jalmari and Rauha Ahokas Foundation. The authors declare that there is no conflict of interest that would prejudice the impartiality of this scientific work.

\section{References}

Adazawa H \& Komuro I 2003 Roles of cardiac transcription factors in cardiac hypertrophy. Circulation Research 92 1079-1088.

Albert CJ \& Ford DA 1998 Identification of specific protein kinase C isoforms and accelerated protein kinase C-dependent nuclear protein phosphorylation during myocardial ischemia. FEBS Letters 438 32-36.

Cao L \& Gardner DG 1995 Natriuretic peptides inhibit DNA synthesis in cardiac fibroblasts. Hypertension 25 227-234.

Day ML, Schwartz D, Wiegand RC, Stockman PT, Brunnert SR, Tolunay HE, Currie MG, Standaert DG \& Needleman P 1987 Ventricular atriopeptin: unmasking of mRNA and peptide synthesis by hypertrophy or dexamethasone. Hypertension 9 485-491.

Du XJ 2004 Gender modulates cardiac phenotype development in genetically modified mice. Cardiovascular Research 63 510-519.

Eklund L, Piuhola J, Komulainen J, Sormunen R, Ongvarrasopone C, Fassler R, Muona A, Ilves M, Ruskoaho H, Takala TE et al. 2001 Lack of type XV collagen causes a skeletal myopathy and cardiovascular defects in mice. PNAS 98 1194-1199. 
Espiner EA, Richards AM, Yandle TG \& Nicholls MG 1995 Natriuretic hormones. Endocrinology and Metabolism Clinics of North America 24 481-509.

Frey N, McKinsey TA \& Olson EN 2000 Decoding calcium signals involved in cardiac growth and function. Nature Medicine 6 1221-1227.

Gusterson RJ, Jazrawi E, Adcock IM \& Latchman DS 2003 The transcriptional co-activators CREB-binding protein (CBP) and p300 play a critical role in cardiac hypertrophy that is dependent on their histone acetyltransferase activity. Journal of Biological Chemistry 278 $6838-6847$.

Holtwick R, van Eickels M, Skryabin BV, Baba HA, Bubikat A, Begrow F, Schneider MD, Garbers DL \& Kuhn M 2003 Pressure-independent cardiac hypertrophy in mice with cardiomyocyte-restricted inactivation of the atrial natriuretic peptide receptor guanylyl cyclase-A. Journal of Clinical Investigation 111 1399-1407.

Horio T, Nishikimi T, Yoshihara F, Matsuo H, Takishita S \& Kangawa K 2000 Inhibitory regulation of hypertrophy by endogenous atrial natriuretic peptide in cultured cardiac myocytes. Hypertension 35 19-24.

Karsai A, Miller S, Platz S \& Hauser MT 2002 Evaluation of a homemade SYBR green I reaction mixture for real-time PCR quantification of gene expression. BioTechniques 32 790-796.

Knowles JW, Esposito G, Mao L, Hagaman JR, Fox JE, Smithies O, Rockman HA \& Maeda N 2001 Pressure-independent enhancement of cardiac hypertrophy in natriuretic peptide receptor A-deficient mice. Journal of Clinical Investigation 107 975-984.

Kuhn M, Holtwick R, Baba HA, Perriad JC, Schmitz W \& Ehler E 2002 Progressive cardiac hypertrophy and dysfunction in atrial natriuretic peptide receptor (GC-A) deficient mice. Heart $\mathbf{8 7}$ 368-374.

Li Y, Kishimoto I, Saito Y, Harada M, Kuwahara K, Izumi T, Takahashi N, Kawakami R, Tanimoto K, Nakagawa Y et al. 2002 Guanylyl cyclase-A inhibits angiotensin II type 1A receptor-mediated cardiac remodelling, an endogenous protective mechanism in the heart. Circulation 106 1722-1728.

Li Y, Kishimoto I, Saito Y, Harada M, Kuwahara K, Izumi T, Hamanaka I, Takahashi N, Kawakami R, Tanimoto K et al. 2004 Androgen contributes to gender-related cardiac hypertrophy and fibrosis lacking the gene encoding guanylyl cyclase-A. Endocrinology 145 951-958.

Lin KF, Chao J \& Chao L 1998 Atrial natriuretic peptide gene delivery attenuates hypertension, cardiac hypertrophy, and renal injury in salt-sensitive rats. Human Gene Therapy 9 1429-1438.

Manabe I, Shindo T \& Nagai R 2002 Gene expression in fibroblasts and fibrosis - involvement in cardiac hypertrophy. Circulation Research 91 1103-1113.
Muller M, van der Schouw YT, Thijssen JH \& Grobbee DE 2003 Endogenous sex hormones and cardiovascular disease in men. Journal of Clinical Endocrinology and Metabolism 88 5076-5086.

Ogawa Y, Tamura N, Chusho H \& Nakao K 2001 Brain natriuretic peptide appears to act locally as an antifibrotic factor in the heart. Canadian Journal of Physiology and Pharmacology 79 723-729.

Oliver PM, Fox JE, Kim R, Rockman HA, Kim HS, Reddick RL, Pandey KN, Milgram SL, Smithies O \& Maeda N 1997 Hypertension, hypertrophy, and sudden death in mice lacking natriuretic peptide receptor A. PNAS 94 14730-14735.

Piuhola J, Szokodi I, Kinnunen P, Ilves M, de Chatel R, Vuolteenaho O \& Ruskoaho H 2003 Endothelin-1 contributes to the Frank-Starling response in hypertrophic rat hearts. Hypertension 41 93-98.

Rosenkranz S, Flesch M, Amann K, Haeuseler C, Kilter H, Seeland U, Schluter K-D \& Bohm M 2002 Alterations of $\beta$-adrenergic signaling and cardiac hypertrophy in transgenic mice overexpressing TGF- $\beta 1$. American Journal of Physiology 283 H1253-H1262.

Simon RM \& Dobbin K 2003 Experimental design of DNA microarray experiments. BioTechniques Suppl 16-21.

Spitaler M, Villunger A, Grunicke H \& Uberall F 2000 Unique structural and functional properties of the ATP-binding domain of atypical protein kinase C-iota. Journal of Biological Chemistry 275 33289-33296.

Stromer H, Cittadini A, Szymanska G, Apstein CS \& Morgan JP 1997 Validation of different methods to compare isovolumic cardiac function in isolated hearts of varying sizes. American Journal of Physiology 272 H501-H510.

Swynghedauw B 1999 Molecular mechanisms of myocardial remodelling. Physiological Reviews 79 215-262.

Swynghedauw B 2006 Phenotypic plasticity of adult myocardium: molecular mechanisms. Journal of Experimental Biology 209 2320-2327.

Tenhunen O, Sarman B, Kerkela R, Szokodi I, Papp L, Toth M \& Ruskoaho H 2004 Mitogen activated protein kinases p38 and ERK1 / 2 mediate the wall stress-induced activation of GATA4 binding in adult heart. Journal of Biological Chemistry 279 24852-24860.

Tokudome T, Horio T, Kishimoto I, Soeki T, Mori K, Kawano Y, Kohno M, Garbers DL, Nakao K \& Kangawa K 2005 Calcineurin-nuclear of activated t cells pathway-dependent cardiac remodelling in mice deficient in guanylyl cyclase A, a receptor for atrial and brain natriuretic peptides. Circulation 111 3095-3104.

Yandle TG, Fitzpatrick MA, Espiner EA, Richards AM, Fisher S \& Carne A 1991 Ovine atrial natriuretic factor - sequence of circulating forms and metabolism in plasma. Peptides 12 279-283.

Zhang CL, McKinsey TA, Chang S, Antos CL, Hill JA \& Olsen EN 2002 Class II histone deacetylases act as signal-responsive repressors of cardiac hypertrophy. Cell 110 479-488. 